\title{
Noninvasive detection of molecular bonds in quantum dots
}

\author{
M. C. Rogge* and R. J. Haug \\ Institut für Festkörperphysik, Leibniz Universität Hannover, Appelstrasse 2, 30167 Hannover, Germany
}

(Received 31 July 2008; published 28 October 2008)

\begin{abstract}
We performed charge detection on a lateral triple quantum dot with starlike geometry. The setup allows us to interpret the results in terms of two double dots with one common dot. One double dot features weak tunnel coupling and can be understood with atomlike electronic states, the other one is strongly coupled forming moleculelike states. In nonlinear measurements we identified patterns that can be analyzed in terms of the symmetry of tunneling rates. Those patterns strongly depend on the strength of interdot tunnel coupling and are completely different for atomiclike or moleculelike coupled quantum dots allowing the noninvasive detection of molecular bonds.
\end{abstract}

DOI: 10.1103/PhysRevB.78.153310

PACS number(s): 73.21.La, 73.23.Hk, 73.63.Kv

Quantum dots are often called artificial atoms ${ }^{1}$ due to their discrete electronic level spectrum. When several quantum dots are connected, they start to interact. ${ }^{2}$ If the tunneling rate between the dots is small, the electronic wave functions are still constricted to the single-quantum dots and the interaction is dominated by electrostatics with sequential interdot tunneling. In contrast, for large tunneling rates electronic states can be found extended over several dots. These extended states introduce covalent bonding as in real molecules. ${ }^{3}$ Whether or not the interdot tunneling rates are sufficient to form coherent moleculelike states is a crucial information in order to properly describe a quantum dot system. Especially for quantum computing purposes ${ }^{4}$ coherent states are necessary to form and couple qubits and to implement SWAP gates for qubit manipulation. 5,6

With time resolved charge detection tunneling rates and coupling strengths can be observed directly (e.g. Ref. 7). With dc-transport experiments, however, there are only a few methods that can give hints for molecular bonds. The width of anticrossings visible in charging diagrams is a measure, ${ }^{8}$ although anticrossings appear for capacitively coupled dots as well. In addition the curvature of the lines forming an anticrossing can be used ${ }^{9}$ and also the visibility of lines in nonparallel quantum dots. ${ }^{10}$ Another alternative is to study excited states. Strongly coupled quantum dots form bonding and antibonding states that are visible in nonlinear measurements. ${ }^{11}$

We have studied the impact of the coupling strength on the mean charge of multiple quantum dot systems coupled in series. Using a quantum point contact $^{12}$ we analyzed the mean charge in stability diagrams and interpret the results in terms of resonant tunneling. We found that depending on the symmetry of tunneling rates and on the coupling strength, characteristic patterns are formed in nonlinear measurements. This allows us to noninvasively detect the symmetry of tunneling rates and the quality of the interdot coupling.

The measurements were performed on a device containing three quantum dots A, B, and C (see inset of Fig. 1). The device was produced using local anodic oxidation on a GaAs/AlGaAs heterostructure. ${ }^{13,14}$ The three dots are positioned in a starlike geometry with one lead for each dot (source S at A, drain1 D1 at B and drain2 D2 at C). The barriers and the potentials can be tuned with four side gates $\mathrm{G} 1$ to G4. A quantum point contact (QPC) biased with $4 \mathrm{mV}$ is placed next to the three dots for charge detection. The device is described in detail in Ref. 15.

Figure 1 shows a charging diagram of the triple dot device as a function of the voltages applied to gates G3 and G1. The derivative of the QPC current with respect to $V_{\mathrm{G} 3}$ is plotted. Dark lines correspond to an increase in charge detected by the QPC (e.g., charging a dot with an electron), bright features appear when the QPC detects a decrease in charge. Three sets of lines are visible that denote charging of the three dots. Lines with a shallow slope correspond to dot B, those with steep slopes appear due to charging of dot $\mathrm{C}$. Intermediate slopes correspond to dot A. Whenever two lines from different sets intersect, an anticrossing appears due to finite coupling between the dots. At these anticrossings, two of the dots are in resonance and can thus be treated as a double quantum dot. From transport measurement we know that the double dots A-B and A-C feature finite interdot tunnel coupling in addition to a certain capacitive coupling, while the double dot B-C is coupled capacitively only (see Ref. 15). Therefore B-C is not interesting for the purpose of this Brief Report. In the following we concentrate on the

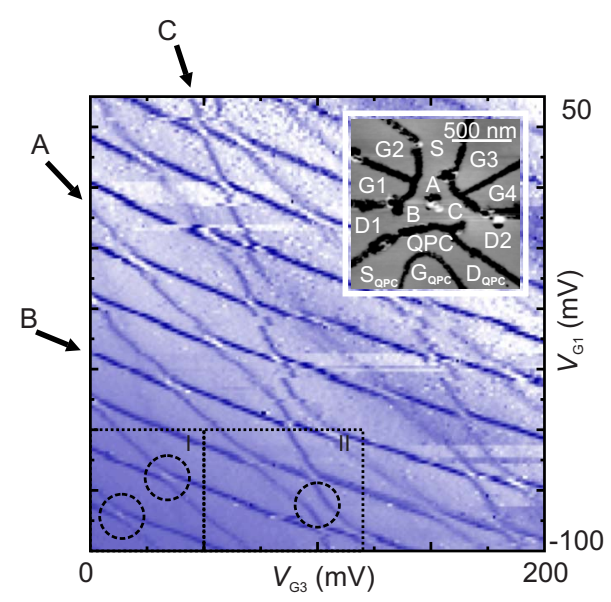

FIG. 1. (Color online) Charging diagram of the triple dot device measured with charge detection. Three sets of lines appear from the three dots (A, B, and C). At the intersections, anticrossings are visible due to interdot coupling (circles). Inset: atomic force microscopic image of the device with dots $\mathrm{A}, \mathrm{B}$, and $\mathrm{C}$ and a quantum point contact for charge detection. 

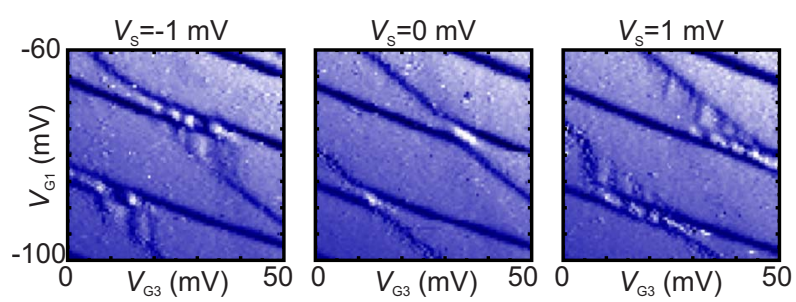

FIG. 2. (Color online) Charge measurement in Sec. I of Fig. 1 for $V_{\mathrm{S}}=-1 \mathrm{mV}, V_{\mathrm{S}}=0 \mathrm{mV}$ and $V_{\mathrm{S}}=1 \mathrm{mV}$. At $V_{\mathrm{S}} \neq 0$ triangular patterns appear with additional lines of dark and bright features due to excited atomic states.

analysis of the two double dots A-B and A-C. This analysis is done in the two sections (Secs. I and II) with Sec. I showing anticrossings due to resonance of dots A and B, Sec. II showing an anticrossing due to resonance of dots $\mathrm{A}$ and $\mathrm{C}$ (circles). In this regime transport through the dots is not measurable anymore as the tunneling rates to the leads are too low.

Figure 2 shows three graphs measured in the region of Sec. I. Charge detection is performed in the linear regime with $V_{\mathrm{S}}=0$ (center image) and in the nonlinear regime with $V_{\mathrm{S}}=-1 \mathrm{mV}$ (left) and $V_{\mathrm{S}}=1 \mathrm{mV}$ (right). While the center image shows the same pattern as observed in Fig. 1 with two sets of lines for dots $\mathrm{A}$ and $\mathrm{B}$ and two anticrossings, the situation changes in the nonlinear regime showing a more complex pattern with ground and excited states. The anticrossings appear shifted to the upper left for $V_{S}=-1 \mathrm{mV}$ and to the lower right for $V_{\mathrm{S}}=1 \mathrm{mV}$. A triangular shaped pattern with additional lines is connected to the right $\left(V_{\mathrm{S}}=-1 \mathrm{mV}\right)$ and to the left, respectively $\left(V_{\mathrm{S}}=1 \mathrm{mV}\right)$. These triangles are familiar from nonlinear transport measurements in weakly coupled quantum dots ${ }^{2,16}$ and have recently been measured with charge detection as well. ${ }^{17}$ However, triangles with such patterns have not been reported so far. Dark and bright features alternate corresponding to alternating increase and decrease in mean charge measured with the QPC. The effect scales linear with $V_{\mathrm{S}}$. Thus back action from the QPC due to the finite QPC bias seems not to be significant.

The origin of this pattern is explained with the schematics shown in Fig. 3. Assuming the total number of electrons to be $N_{\mathrm{A}}-1$ or $N_{\mathrm{A}}$ on dot $\mathrm{A}$ and $N_{\mathrm{B}}-1$ or $N_{\mathrm{B}}$ on dot B with ground-state energies $E_{N \mathrm{~A}-1}$ or $E_{N \mathrm{~A}}$ and $E_{N \mathrm{~B}-1}$ or $E_{N \mathrm{~B}}$, the following two transitions are possible:

$$
E_{N \mathrm{~A}-1} \leftrightarrow E_{N \mathrm{~A}}
$$

with chemical potential

$$
\begin{gathered}
\mu_{N \mathrm{~A}}=E_{N \mathrm{~A}}-E_{N \mathrm{~A}-1}, \\
E_{N \mathrm{~B}-1} \leftrightarrow E_{N \mathrm{~B}}
\end{gathered}
$$

with chemical potential

$$
\mu_{N \mathrm{~B}}=E_{N \mathrm{~B}}-E_{N \mathrm{~B}-1} .
$$

If these chemical potentials equal those of the leads $\left(\mu_{\mathrm{S}}\right.$ and $\mu_{\mathrm{D} 1}$ ), lines are visible in the charging diagram. At $V_{\mathrm{S}}=0$ (left schematic) $\mu_{\mathrm{S}}$ and $\mu_{\mathrm{D} 1}$ are degenerate. Therefore each chemical dot potential produces a single line forming the
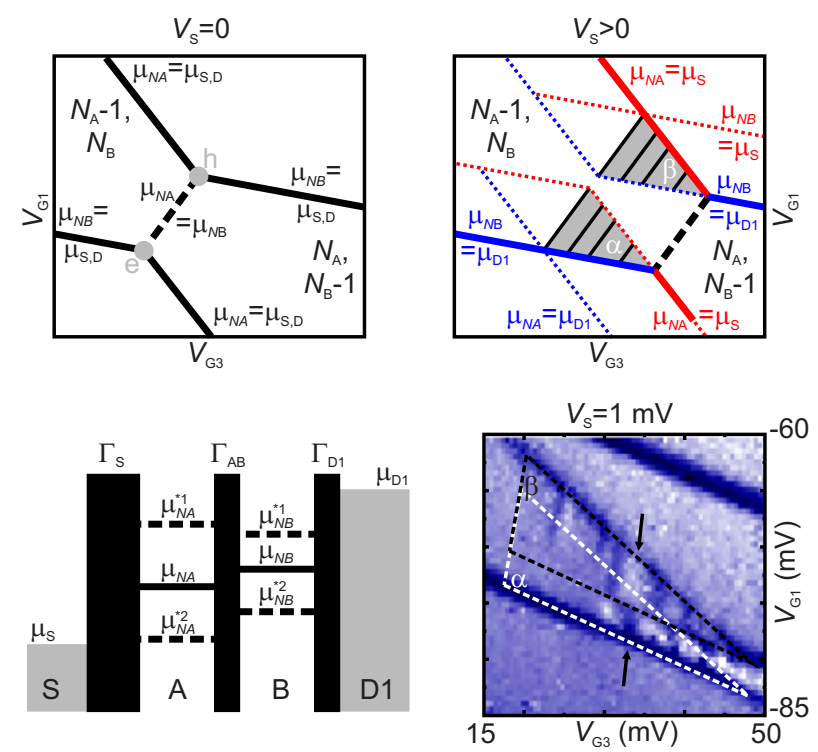

FIG. 3. (Color online) Top left: schematic for the anticrossing of dots $\mathrm{A}$ and $\mathrm{B}$ for $V_{\mathrm{S}}=0$ with triple points $e$ and $h$. Top right: schematic for the anticrossing at $V_{\mathrm{S}}>0$. Two triangles $(\alpha$ and $\beta$ ) appear with additional lines. Bottom left: possible configuration of chemical potentials. Bottom right: section of Fig. $2, V_{S}=1 \mathrm{mV}$. The two triangles $(\alpha$ and $\beta)$ are visible even though they overlap. The line pattern has the opposite order in both triangles. A dark line in $\alpha$ becomes bright in $\beta$.

typical hexagonal cells with the so-called triple points (marked with $e$ and $h$ ) at the edges. At $e$ transport through the serial double quantum dot can be described by sequential tunneling of one electron at a time through the otherwise empty dot states. At $h$ transport occurs by sequential tunneling of one hole at a time through the otherwise filled dot states. In between the two triple points the chemical potentials of both dots are equal $\left(\mu_{N \mathrm{~A}}=\mu_{N \mathrm{~B}}\right)$ and an electron can move from dot B to dot A with increasing $V_{\mathrm{G} 3}$. As dot A is further away from the QPC, the QPC detects a decrease in charge. Thus a white feature is visible at the anticrossings in the center image of Fig. 2.

The nonlinear regime is described with the schematic on the right $\left(V_{\mathrm{S}}>0\right)$. The discussion for $V_{\mathrm{S}}<0$ is analog. At $V_{\mathrm{S}}>0$ the degeneracy of $\mu_{\mathrm{S}}$ and $\mu_{\mathrm{D} 1}$ is lifted, $\mu_{\mathrm{D} 1}>\mu_{\mathrm{S}}$. Therefore there are two possible resonance conditions for each chemical dot potential. But as each dot does only couple to one lead, only one resonance condition per dot is relevant. Thus still only one line is visible per ground-state transition (with $\mu_{N \mathrm{~A}}=\mu_{\mathrm{S}}$ and $\mu_{N \mathrm{~B}}=\mu_{\mathrm{D} 1}$ ). The other resonance conditions do not appear (dotted lines). As the two dots use different chemical lead potentials the exchange of an electron between the dots might not appear at $\mu_{N \mathrm{~A}}=\mu_{N \mathrm{~B}}$ but shifted to the lower right at the dashed black line. This depends on the ratio of interdot relaxation and cotunneling rates. Left to this line there are two triangles (gray) where both chemical potentials, $\mu_{N \mathrm{~A}}$ and $\mu_{N \mathrm{~B}}$, are between $\mu_{\mathrm{S}}$ and $\mu_{\mathrm{D} 1}$ and $\mu_{N \mathrm{~A}}<\mu_{N \mathrm{~B}}$ (Fig. 3, bottom left). These are the triangles described above. As observed in the right image of Fig. 2 they appear shifted to the lower right compared to the anticrossing at $V_{\mathrm{S}}=0$. At the left border of these triangles the resonance condition $\mu_{N \mathrm{~A}}=\mu_{N \mathrm{~B}}$ is fulfilled opening a trans- 
port channel. Further transport channels within the triangles can appear due to excited atomic states.

As an example we take into account two excited states per dot with total energies $E_{N-1}^{*}>E_{N-1}$ and $E_{N}^{*}>E_{N}$. Now the following two additional transitions are possible for each dot with new chemical potentials (Fig. 3, bottom left):

$$
E_{N-1} \leftrightarrow E_{N}^{*}
$$

with chemical potential

$$
\begin{aligned}
& \mu_{N}^{* 1}>\mu_{N}, \\
& E_{N-1}^{*} \leftrightarrow E_{N}
\end{aligned}
$$

with chemical potential

$$
\mu_{N}^{* 2}<\mu_{N}
$$

Additional transport channels form for $V_{\mathrm{S}}>0$, if the following resonance conditions are fulfilled (other channels are forbidden due to trapping):

$$
\begin{aligned}
& \mu_{N \mathrm{~A}}=\mu_{N \mathrm{~B}}^{* 2}, \\
& \mu_{N \mathrm{~A}}^{* 1}=\mu_{N \mathrm{~B}}, \\
& \mu_{N \mathrm{~A}}^{* 1}=\mu_{N \mathrm{~B}}^{* 2} .
\end{aligned}
$$

Together with the resonance condition $\mu_{N \mathrm{~A}}=\mu_{N \mathrm{~B}}$ those are the four solid lines drawn in each gray triangle in the schematic. They can appear in conductance measurements with electronlike transport in triangle $\alpha$ and holelike transport in triangle $\beta$.

With charge detection, resonances are only visible if they feature a different mean charge than what is given in the gray regions. Within the gray triangles at $V_{\mathrm{S}}>0$ electrons can enter dot B via drain1, holes can enter dot A via source (electrons can leave A). Off resonance no transport between the dots is possible. Therefore the mean charge in the gray regions, added to the charge background of $N_{\mathrm{A}}$ and $N_{\mathrm{B}}$ electrons, is one electron on dot $\mathrm{B}$. On resonance, transport between the dots is possible. Now the mean charge depends on the symmetry of the tunneling rates $\Gamma_{S}$ between source and $\operatorname{dot} \mathrm{A}, \Gamma_{\mathrm{D} 1}$ between drain 1 and $\operatorname{dot} \mathrm{B}$, and the interdot tunneling rate $\Gamma_{\mathrm{AB}}$. The following three different symmetries are possible that define the mean charge on resonance within triangles $\alpha$ and $\beta$ :

(i) $\Gamma_{\mathrm{AB}}<\Gamma_{\mathrm{S}}, \Gamma_{\mathrm{D} 1}$ :

$\alpha$ : one electron on $\mathrm{B}$.

$\beta$ : one electron on $\mathrm{B}$.

(ii) $\Gamma_{\mathrm{D} 1}<\Gamma_{\mathrm{S}}, \Gamma_{\mathrm{AB}}$ :

$\alpha$ : no electron on $\mathrm{A}$ and $\mathrm{B}$.

$\beta$ : one electron equally occupying both dots.

(iii) $\Gamma_{\mathrm{S}}<\Gamma_{\mathrm{AB}}, \Gamma_{\mathrm{D} 1}$ :

$\alpha$ : one electron equally occupying both dots.

$\beta$ : one electron on both dots each.

In (i) no lines are visible with charge detection as the mean charge on resonance is identical to the mean charge off resonance in the gray regions. In (ii) and (iii) a resonance changes the mean charge in both triangles and becomes visible. Thus it is much more probable to observe excited states

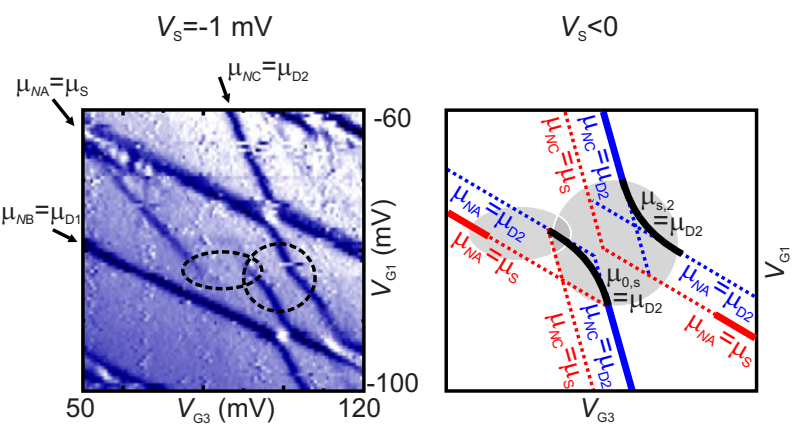

FIG. 4. (Color online) Left: Sec. II from Fig. 1 at $V_{\mathrm{S}}=-1 \mathrm{mV}$. An anticrossing of dots $\mathrm{A}$ and $\mathrm{C}$ is visible (circle). Instead of triangular shaped patterns a splitting is observed on the left (ellipse). Right: schematic for the measurement on the left. The pattern is formed by a molecular state that is created at the anticrossing.

in weakly coupled double dots than in single dots, where excited states can only appear with symmetric tunneling rates. $^{18}$

Therefore (ii) or (iii) must be true for the measurements presented in Fig. 2. A more detailed analysis reveals the actual ratio of tunneling rates. The bottom of Fig. 3 shows a section of the right image in Fig. 2 with the triangles $\alpha$ and $\beta$ marked with white and black lines. As the difference between $\mu_{\mathrm{S}}$ and $\mu_{\mathrm{D} 1}$ is bigger than the splitting of the anticrossing, both triangles overlap. Within the triangles the additional lines are visible. Following the line marked with arrows from bottom to top, one first observes a dark feature in triangle $\alpha$ and then a bright feature in triangle $\beta$. Thus the effect on the mean charge must be vice versa in both triangles. This is only possible in (iii) with a decrease in mean charge in (iii) $\alpha$ (as dot B is closer to the QPC than $\operatorname{dot} \mathrm{A}$ ) and an increase in (iii) $\beta$.

Within the overlapped region an electron can enter the possibly empty dots via drain1, as the system is within triangle $\alpha$. This electron can now leave the dots again via source, or another electron can enter via drain1, as the system is in triangle $\beta$ as well. As $\Gamma_{\mathrm{S}}<\Gamma_{\mathrm{D} 1}$, it is much more probable for a second electron to enter the system than for the first one to leave. Therefore the process related to triangle $\beta$ is favored.

Triangular patterns are visible for A-B over a wide range of parameters. They finally fade out with increasing gate voltages as the tunneling rates are changed. In contrast no such patterns appear for A-C, although measured under the same conditions within the same device. Instead a different pattern is found. The left of Fig. 4 shows a measurement at $V_{\mathrm{S}}=-1 \mathrm{mV}$, taken within Sec. II (as the lines of dot A appear steeper than those of $\mathrm{B}$, but shallower than those of $\mathrm{C}$, patterns of A-B at $V_{\mathrm{S}}>0$ must be compared with patterns of A-C with $\left.V_{\mathrm{S}}<0\right)$. The measurement shows an anticrossing (circle) that is almost not shifted compared to the one observed at $V_{S}=0$ (see Fig. 1). Another striking feature is the step that appears on the left of the anticrossing (ellipse). The left line of the anticrossing disappears and comes up again with a huge offset to the left. There are no triangular shaped patterns or lines for excited states.

The origin of this pattern is described using the schematic 
at the right of Fig. 4 assuming molecular bonds. With a relative width of anticrossings of $\approx 0.4$ (with 1 being the maximum $^{8}$ ) the double dot A-C is coupled stronger than A-B, which has a relative width of ca. 0.33. As for the schematics shown before for $V_{\mathrm{S}} \neq 0$ the resonances for groundstate transitions split into two resonances as the chemical potentials in the leads differ. Here with $V_{\mathrm{S}}<0$ resonances with $\mu_{\mathrm{S}}$ appear shifted to the lower left compared to those with $\mu_{\mathrm{D} 2}$. Far off the anticrossing states of the two dots can be described as atomic with chemical potentials $\mu_{N \mathrm{~A}}$ and $\mu_{N C}$. As dot $\mathrm{A}$ is coupled to source and $\mathrm{C}$ to drain2, the resonances $\mu_{N \mathrm{~A}}=\mu_{\mathrm{S}}$ and $\mu_{N \mathrm{C}}=\mu_{\mathrm{D} 2}$ must appear (solid straight lines). Due to the strong coupling of $\mathrm{A}$ and $\mathrm{C}$ the pattern around the anticrossing cannot be described with atomic states any longer. Instead a common symmetric molecular state with energy $E_{\mathrm{s}}$ evolves that is extended over the whole double dot. With the double dot energies $E_{0}$ for no added electrons and $E_{2}$ for two electrons added, the following two new transitions are possible:

$$
E_{0} \leftrightarrow E_{\mathrm{s}}
$$

with chemical potential

$$
\begin{gathered}
\mu_{0, \mathrm{~s}}<\mu_{N \mathrm{~A}}, \mu_{N \mathrm{C}}, \\
E_{\mathrm{s}} \leftrightarrow E_{2}
\end{gathered}
$$

with chemical potential

$$
\mu_{\mathrm{s}, 2}>\mu_{N \mathrm{~A}}, \mu_{N \mathrm{C}}
$$

These new chemical potentials can create two resonances each, one with $\mu_{\mathrm{S}}$, one with $\mu_{\mathrm{D} 2}$. Which of those involves a change in the mean charge depends on the tunneling rates again. If $\Gamma_{\mathrm{D} 2}<\Gamma_{\mathrm{S}}$, charging appears at resonance with $\mu_{\mathrm{S}}$. If $\Gamma_{\mathrm{S}}<\Gamma_{\mathrm{D} 2}$, charging appears at resonance with $\mu_{\mathrm{D} 2}$ instead. The latter case results in the two curved solid lines in the schematic, which properly describes the experiment. In the area close to the anticrossing the double dot shows charging at resonance with $\mu_{\mathrm{D} 2}$ as well as dot $\mathrm{C}$ far off the anticrossing. Dot $\mathrm{A}$ shows charging at resonance with $\mu_{\mathrm{S}}$ instead. Therefore a jump must occur when the system changes from the molecular common state to the atomic state of dot A. Two of those jumps are shown in the schematic but only one is visible in the measurement as the other one is disturbed by a line of dot B. However, the symmetry of tunneling rates is detected for double dot A-C as well. The antibonding state is not visible probably due to the strength of tunneling asymmetry that can only be compensated up to a certain amount by detuning the double dot, as shown in noninvasive measurements of molecular states by Hüttel et al. ${ }^{11}$ However, it would appear at the lower right where a line of B crosses.

Thus with charge measurements it is possible to detect the symmetry of tunneling rates for weakly and for strongly coupled double quantum dots. For the two double dots in this device the same symmetry was detected: $\Gamma_{\mathrm{S}}<\Gamma_{\mathrm{AB}, \mathrm{AC}}$, $\Gamma_{\mathrm{D} 1, \mathrm{D} 2}$. However, depending on the strength of tunnel coupling two completely different patterns were found. Thus noninvasive charge measurement is capable of detecting molecular bonds in quantum dots.

For the heterostructure we thank M. Bichler, G. Abstreiter, and W. Wegscheider. This work has been supported by BMBF via nanoQUIT. *rogge@nano.uni-hannover.de

${ }^{1}$ L. P. Kouwenhoven, C. M. Marcus, P. L. McEuen, S. Tarucha, R. M. Westervelt, and N. S. Wingreen, in Mesoscopic Electron Transport, NATO Advanced Study Institute, Series E: Applied Science, edited by L. L. Sohn, L. P. Kouwenhoven, and G. Schön (Kluwer, Dordrecht, 1997), Vol. 345, pp. 105-214.

${ }^{2}$ W. G. van der Wiel, S. D. Franceschi, J. M. Elzerman, T. Fujisawa, S. Tarucha, and L. P. Kouwenhoven, Rev. Mod. Phys. 75, 1 (2002).

${ }^{3}$ R. H. Blick, R. J. Haug, J. Weis, D. Pfannkuche, K. v. Klitzing, and K. Eberl, Phys. Rev. B 53, 7899 (1996).

${ }^{4}$ D. Loss and D. P. DiVincenzo, Phys. Rev. A 57, 120 (1998).

${ }^{5}$ J. R. Petta, A. C. Johnson, J. M. Taylor, E. A. Laird, A. Yacoby, M. D. Lukin, C. M. Marcus, M. P. Hanson, and A. C. Gossard, Science 309, 2180 (2005).

${ }^{6}$ R. Hanson, L. P. Kouwenhoven, J. R. Petta, S. Tarucha, and L. M. K. Vandersypen, Rev. Mod. Phys. 79, 1217 (2007).

${ }^{7}$ S. Gustavsson, M. Studer, R. Leturcq, T. Ihn, K. Ensslin, D. C. Driscoll, and A. C. Gossard, Phys. Rev. Lett. 99, 206804 (2007).

${ }^{8}$ J. M. Golden and B. I. Halperin, Phys. Rev. B 54, 16757 (1996).
${ }^{9}$ R. H. Blick, D. Pfannkuche, R. J. Haug, K. v. Klitzing, and K. Eberl, Phys. Rev. Lett. 80, 4032 (1998).

${ }^{10}$ M. C. Rogge, C. Fühner, U. F. Keyser, and R. J. Haug, Appl. Phys. Lett. 85, 606 (2004).

${ }^{11}$ A. K. Hüttel, S. Ludwig, H. Lorenz, K. Eberl, and J. P. Kotthaus, Phys. Rev. B 72, 081310(R) (2005).

${ }^{12}$ M. Field, C. G. Smith, M. Pepper, D. A. Ritchie, J. E. F. Frost, G. A. C. Jones, and D. G. Hasko, Phys. Rev. Lett. 70, 1311 (1993).

${ }^{13}$ M. Ishii and K. Matsumoto, Jpn. J. Appl. Phys., Part 1 34, 1329 (1995).

${ }^{14}$ U. F. Keyser, H. W. Schumacher, U. Zeitler, R. J. Haug, and K. Eberl, Appl. Phys. Lett. 76, 457 (2000).

${ }^{15}$ M. C. Rogge and R. J. Haug, Phys. Rev. B 77, 193306 (2008).

${ }^{16}$ D. Dixon, L. P. Kouwenhoven, P. L. McEuen, Y. Nagamune, J. Motohisa, and H. Sakaki, Phys. Rev. B 53, 12625 (1996).

${ }^{17}$ A. C. Johnson, J. R. Petta, C. M. Marcus, M. P. Hanson, and A. C. Gossard, Phys. Rev. B 72, 165308 (2005).

${ }^{18}$ M. C. Rogge, B. Harke, C. Fricke, F. Hohls, M. Reinwald, W. Wegscheider, and R. J. Haug, Phys. Rev. B 72, 233402 (2005). 